\title{
Correction of Skeletal Class III by Wunderer's Activator and Face Mask
}

\author{
Mahadia Saad Allafe, Marwa S. Shamaa, Maher Abd El-Salam Fouda \\ Department of Orthodontic, Faculty of Dentistry, Mansoura University, Mansoura, Egypt
}

Email for correspondence: msaad1984@yahoo.com

\begin{abstract}
Context: Treatment of skeletal Class III malocclusion in growing patients is a challenging part of contemporary orthodontic practice. Many appliances are now available for correcting skeletal Class III malocclusion due to maxillary deficiency. In the current study, the Wunderer's activator and face mask were used for the correction of skeletal Class III in growing patients to overcome the latter side effects of other appliances. Objective: Evaluation of dentoskeletal changes before and after growth modification by Wunderer's activator and face mask. Materials and Methods: A total of 12 growing Class III subjects with maxillary retrusion in the early mixed or early permanent dentition (6 male and 6 female) were included in this study. The samples were treated by Wunderer's activator and a face mask for 12 months. Lateral cephalometric tracings were done before and after correction to evaluate the efficacy of Wunderer's activator and face mask. Result: The results showed a highly statistically significant increase in the sella-nasion A angle by $1.98(P<0.001)$ and a statistically significant decrease in the sella-nasion B angle by $0.83(P<0.001)$. Furthermore, the wits appraisal was found to be highly significant increased by $2.8 \mathrm{~mm}$. The vertical measurements showed significant increase where the SN/occlusal plane (OCP) angle and the SN/Gonion-Gnathion angle where increased by 2.1 and 1.78 , respectively. The overjet and proclination maxillary incisors display were improved with retroclination of the lower incisors. Conclusion: Face mask and Wunderer's activator were effective in the treatment of skeletal Class III malocclusion due to maxillary retrusion.
\end{abstract}

Key words: Face mask, skeletal Class III, Wunderer's activator

\section{INTRODUCTION}

Treatment of skeletal Class III malocclusion in growing patients is a challenging part of contemporary orthodontic practice. The etiology of skeletal Class III malocclusion is believed to be mainly hereditary, but environmental factors such as mouth breathing and habits may also play a role. ${ }^{[1,2]}$ Multiple studies have documented that the prevalence of angle Class III malocclusion varies greatly within different geographic regions and races. ${ }^{[3]}$

Skeletal Class III malocclusion may result from: (1) Maxillary retrusion, (2) mandibular protrusion,

\begin{tabular}{|l|l|}
\hline Quick Response Code & Article Info: \\
\hline doi: 10.5866/2017.9.10203 & $\begin{array}{l}\text { Received: 04-12-2017 } \\
\text { Revised: } 28-12-2018 \\
\text { Accepted: 05-02-2018 } \\
\text { Available Online: } 15-02-2018,2018 \text { (www. } \\
\text { nacd.in)@ NAD, } 2018 \text { - All rights reserved }\end{array}$ \\
\hline
\end{tabular}

or (3) combined maxillary retrusion and mandibular protrusion. McNamara and Ellis found that $65-67 \%$ of Class III malocclusions were characterized by maxillary retrusion. ${ }^{[4]}$

The timing of correction of skeletal Class III malocclusion is critical for optimum results. The typical age for a successful maxillary protraction treatment is said to be before the age of 10 years. Franchi et al. found that the greatest orthodontic effect was in the early mixed dentition (about the age of 8 years old) versus later stages in skeletal growth and dental. However, patients treated in late mixed dentition still benefited. ${ }^{[4-6]}$

Many appliances are now available for correcting skeletal Class III malocclusion due to maxillary deficiency. In 1970, popularized the concept of correcting maxillary protraction with face mask. ${ }^{[7]}$ In 1983 , modified the Delaire mask by increasing the amount of force generated by the appliance. ${ }^{[8]}$ 
Orthopedic treatment of Class III malocclusion has been described utilizing a Delaire, Petit style face mask, and reverse headgear for maxillary deficiency. ${ }^{[9]}$ Many of the literature showed that many of the treatment approaches can be found in regarding orthodontic and orthopedic treatment of Class III malocclusion including reverse twin block, modified tandem appliance, and two-piece magnetic appliance. ${ }^{[10-12]}$

In the current study, the Wunderer's activator and face mask were used for the correction of skeletal Class III in growing patients to overcome the latter side effects of these appliances. The research hypothesis assumed that no significant difference exists between the Wunderer's activator with a face mask and other appliances.

\section{MATERIALS AND METHODS}

A total of 12 growing Class III patients were selected from the clinic of the orthodontic department, faculty of dentistry. The cases involved in this study had following criteria:(1) Early mixed or early permanent dentition at the start of treatment characterized by a wits appraisal of $-1 \mathrm{~mm}$ or less, (2) skeletal Class III due to maxillary deficiency, (3) reverse overjet and reverse overbite, (4) no cleft and other craniofacial anomaly, and (5) no previous orthodontic treatment.

Control group was not taken in the present study for ethical purposes to avoid exposing the patient to extra radiation without the benefits of treatment. All orthodontic records were taken for every case. Lateral cephalograms, photography, and models were prepared before and after treatment changes produced by Wunderer's activator and face mask.

The cases and their parents were informed about the study and the steps of treatment, and the parents signed consent forms. In addition, assent forms were required from the children who participated in the study.

For each patient, good upper and lower silicon rubber base impressions were taken and bite construction was registered using exactobite in the most retruded of position the mandible. The bite was transferred to the working upper and lower models, articulated in the hinge articulator and used for appliance fabrication.

Wunderer's activator is a removable appliance composed of Adams clasp made of $0.9 \mathrm{~mm}$ stainless steel (SS) wire on the permanent first molars and short labial bow made of $0.8 \mathrm{~mm}$ SS wire were constructed on anterior incisor teeth of both casts. All the components were waxed and a separating medium was applied to the upper and lower casts. The acrylic part was split horizontally with the upper and lower portions connected by Weise screw [Figure 1]. Part of the Weise screw was embedded in the mandibular portion of the activator and the other part of the screw was attached to the maxillary portion of the activator [Figure 2]. Acrylic base plate was made in the upper and lower casts to connect the components of the appliance with a posterior bite plane in between [Figure 3]. Wunderer's activator had two hooks, one on each side in the upper area of the activator at the premolar areas roughly $15 \mathrm{~mm}$ gingival to OCPs for the attachment of elastics

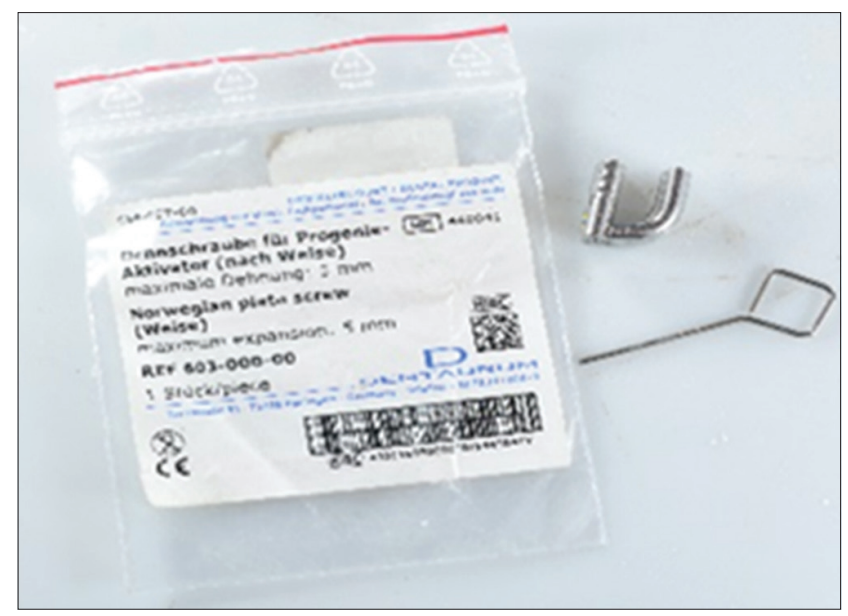

Figure 1: Weise screw

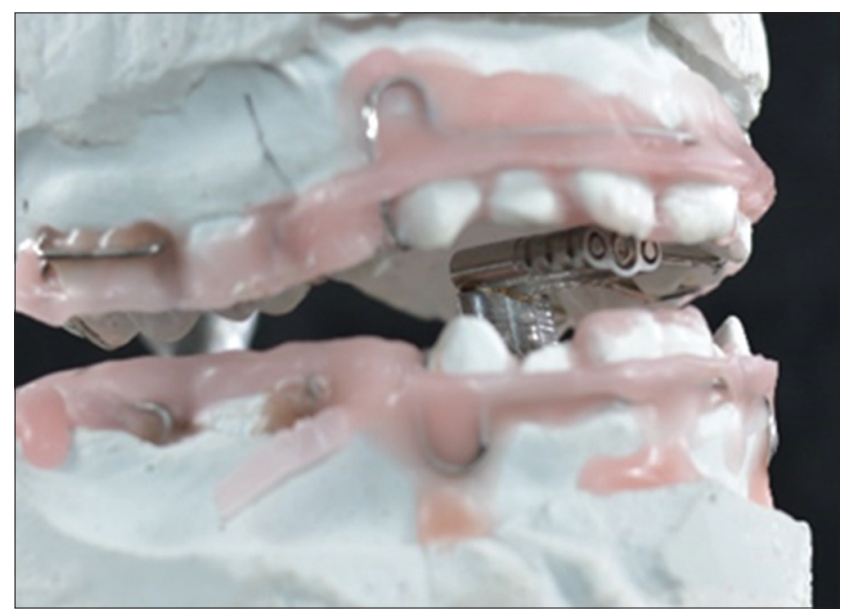

Figure 2: Screw is attached to the maxillary portion of the activator 
[Figure 4], trimming and polishing of the appliance were done to finish it [Figure 5].

\section{Face mask}

Delaire type face mask was attached to the hooks of Wunderer's activator. The face mask was inserted [Figure 6], and an orthopedic force of at least $500 \mathrm{~g}$ per side directed 30 downward and forward from the OCP was applied by heavy extraoral elastics (Ormco, Orange, Calif). The force was measured by a force gauge (Correx Tension Gauge; Haag-Streit Diagnostics, Koeniz, Switzerland). Instructions during the study period: The patients included in the study were instructed to wear the device for a minimum $14 \mathrm{~h}$ every day including the sleeping time. Intraoral appliance had to be removed and cleaned with

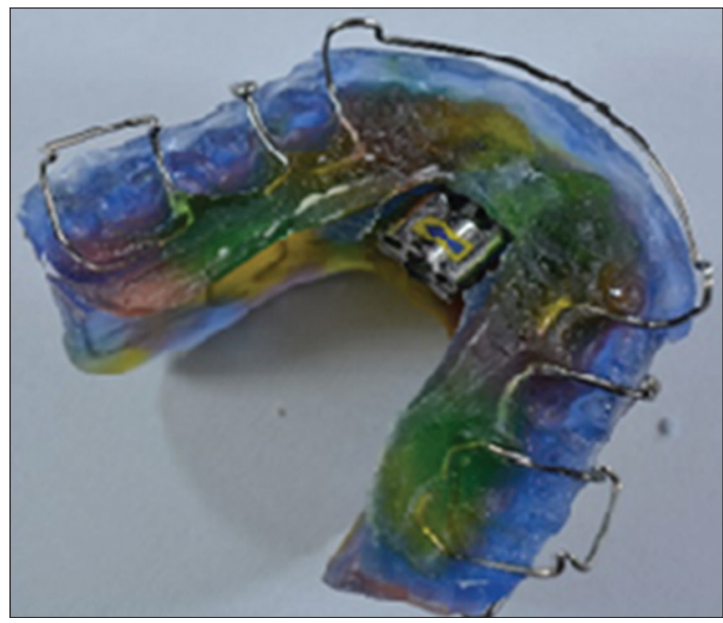

Figure 3: Acrylic base plate was made in the upper and lower casts to connect the components of the appliance with a posterior bite plane in between

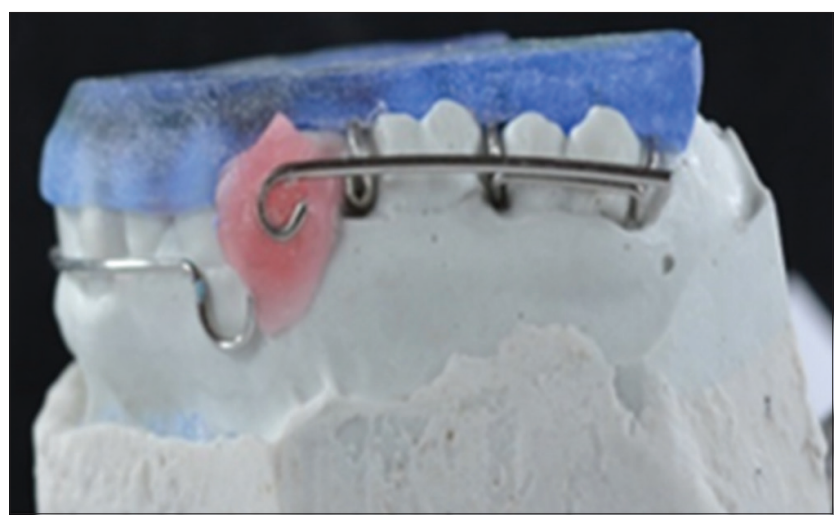

Figure 4: Two hooks one on each side of the upper part of Wunderer's activator, roughly $15 \mathrm{~mm}$ gingival to occlusal planes for the attachment of elastics normal water and mouth cleaning every day to avoid bad odor.

Follow-up was done every 3 weeks, but if there was damage of any part of the appliance or it became unfitted, back directly to the clinic should be done. The elastics were changed every day. Activation of the appliance was achieved by turning the screw using the screwdrivers $1 / 4$ turn twice a week.

All cephalometric radiographs were scanned and analyzed by one investigator using Dolphin Software (Version 11.7; Dolphin Imaging and Management Solutions, Chatsworth, Calif). The analysis consisted of the following:

Angular measurements (sympathetic nerve activity [SNA], sella-nasion B [SNB], ANB, SN/OCP,

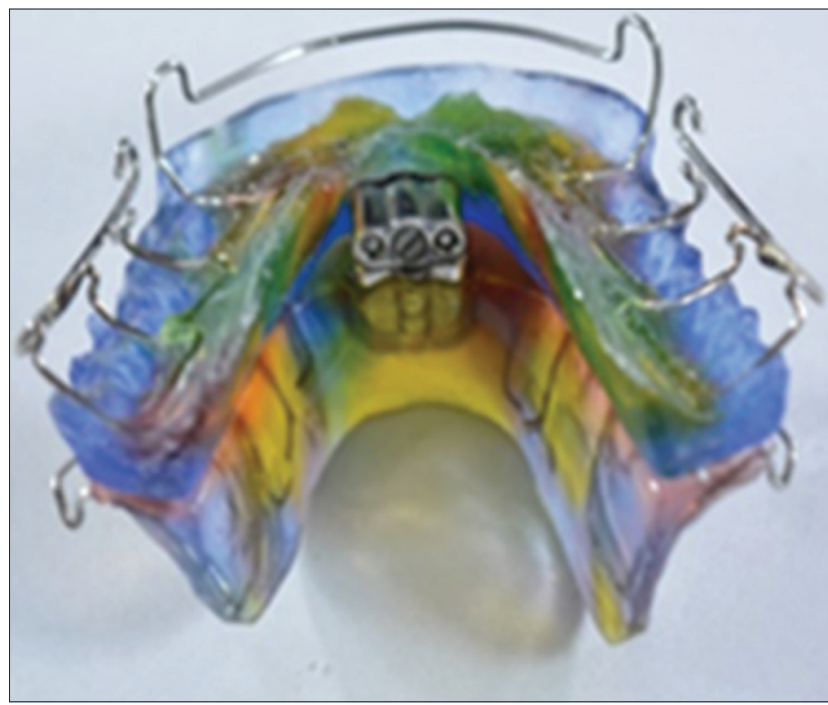

Figure 5: Wunderer's activator

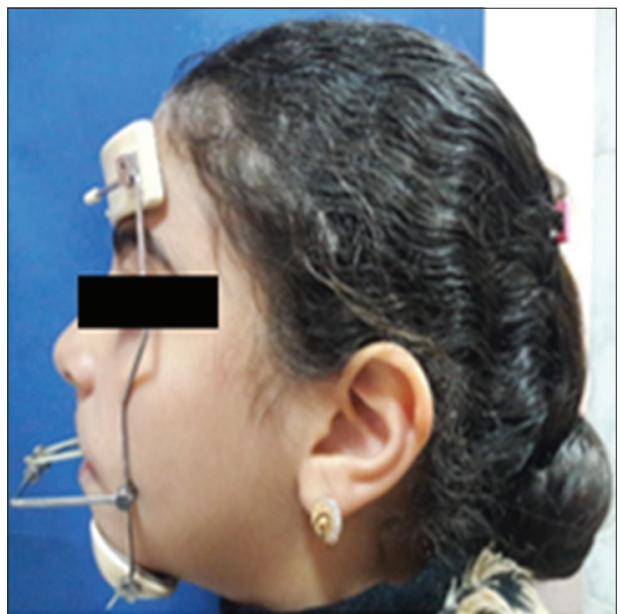

Figure 6: Delaire face mask

Indian J Dent Adv 2018; 9(4): 203-209 
SN/GoGn, SN-U1, U1-NA, U1-palatal plane, L1-NB, L1 mandibular plane [MP], U6-SN, U6-PP, and U6$\mathrm{MP}$ ) and linear measures (U1-NA, L1-NB) [Figure 7].

After correction of the overbite and overjet, the appliance acted as a retainer. After growth modification, correction to start the second phases of treatment for relief any dental malocclusion.

\section{Statistical analysis}

Date from cephalometric radiographs was examined using IBM SPSS Program Version 20.0 90. Quantitative data were described using standard deviation, mean and after testing normality using Shapiro-Wilk test. Significance of the obtained results was judged at the $5 \%$ level and highly statistically significant at $P<0.01$. The used tests were paired $t$-test for quantitative parametric variable, to compare between before and after treatment measures with calculation of mean difference by subtracting post-treatment results minus pre-treatment results.

\section{RESULT}

A total of 12 participiates of both genders (six boys and six girls of the sample) used the Wunderer's activator and a face mask with average age of 8.50 \pm 2.35 years. The data in Table 1 showed that the
SNA angle was highly significant increased by 1.98. Anterior movement of a point was confirmed by the highly statistically significant increase of the

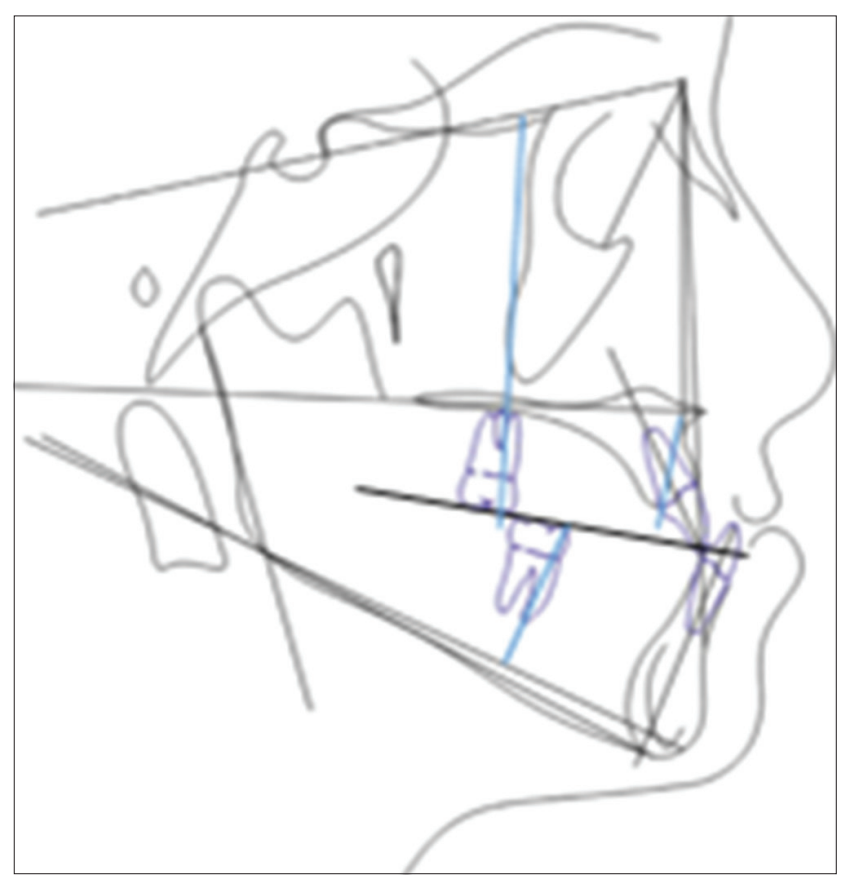

Figure 7: Linear and angular cephalometric measurements Table 1: The present study showed the following linear and angular cephalometric measurements

\begin{tabular}{|c|c|c|c|c|c|}
\hline \multirow[t]{2}{*}{ Measurements } & \multicolumn{2}{|c|}{ Mean \pm SD } & \multirow[t]{2}{*}{ Mean change } & \multirow[t]{2}{*}{ Paired $t$-test } & \multirow[t]{2}{*}{$P$ value } \\
\hline & Pre-treatment & Post-treatment & & & \\
\hline SNA (o) & $80.19 \pm 1.7$ & $82.17 \pm 2.6$ & 1.98 & 5.29 & $0.001 * *$ \\
\hline $\mathrm{SNB}(0)$ & $80.92 \pm 1.8$ & $80.09 \pm 1.9$ & 0.83 & 6.2 & $<0.001 * *$ \\
\hline $\operatorname{ANB}(0)$ & $0.78 \pm 1.13$ & $2.08 \pm 1.5$ & 2.86 & 6.8 & $<0.001 * *$ \\
\hline WITTS (mm) & $5.27 \pm 1.7$ & $2.46 \pm 1.6$ & 2.8 & 6.6 & $<0.001^{* *}$ \\
\hline SN/GOGN (o) & $33.42 \pm 2.96$ & $17 \pm 3.44$ & 1.75 & 4.28 & $0.05^{*}$ \\
\hline $\mathrm{SN} / \mathrm{OCP}(\mathrm{o})$ & $16.64 \pm 3.5$ & $18.65 \pm 2.7$ & 2.01 & 2.76 & $0.05^{*}$ \\
\hline$+1 / \mathrm{NA}(\mathrm{o})$ & $21.4 \pm 4.8$ & $23.86 \pm 3.7$ & 2.5 & 3.6 & $0.007^{* *}$ \\
\hline$+1 / \mathrm{SN}(\mathrm{o})$ & $102.47 \pm 5.8$ & $105.68 \pm 4$ & 2.9 & 2.9 & $0.02^{*}$ \\
\hline 1/NB (o) & $22.82 \pm 4.5$ & $19.14 \pm 1.3$ & 3.7 & 2.7 & $0.028^{*}$ \\
\hline$+1 \mathrm{i} / \mathrm{NA}(\mathrm{mm})$ & $2.30 \pm 1.5$ & $3.27 \pm 1.5$ & 0.97 & 5.5 & $0.001 * *$ \\
\hline $1 \mathrm{i} / \mathrm{NB}(\mathrm{mm})$ & $4.16 \pm 1.4$ & $2.91 \pm 1.4$ & 1.24 & 4.52 & $0.002 * *$ \\
\hline L1/MP (०) & $87.95 \pm 3.07$ & $84.51 \pm 4.4$ & 3.4 & 3.6 & $0.007 * *$ \\
\hline U6/SN (o) & $70.32 \pm 7.02$ & $72.79 \pm 4$ & 2.47 & 1.01 & 0.3 \\
\hline $\mathrm{U6}$ /PP (o) & $78.19 \pm 7.6$ & $79.48 \pm 4.7$ & 1.3 & 0.65 & 0.5 \\
\hline L6/MP ( ) & $82.22 \pm 5.2$ & $78.31 \pm 11$ & 3.91 & 0.95 & 0.37 \\
\hline
\end{tabular}

Comparing between before and after treatment*Statistically significant at $P \leq 0.05$. $* *$ Highly statistically significant $P \leq 0.01$. SD: Standard deviation, SNA: Sella-nasion ASNB: Sella-nasion B, GOGN: Gonion-Gnathion, OCP: Occlusal plane 
ANB angle by $2.86(P<0.001)$. Furthermore, the wits appraisal was found to be highly significant increased by $2.8 \mathrm{~mm}$. On the other hand, statistically significant decrease in the SNB angle was detected by $0.83(P<0.001)$. Regarding the vertical results in the present study showed significant increase in SN-OCP and SN- Gonion-Gnathion (GOGN) by 2.01 and 1.75 , respectively. Regarding the linear and angular dentoalveolar changes in this work, the maxillary incisors proclination was highly significant increased and mandibular incisors inclination was significantly decreased. The U1/ NA, L1/NB, U1-SN, U1/pp, and L1/MP recorded ( $p$ $<0.007,0.28,0.02,0.046$, and 0.007 , respectively). The U6/SN, U6/PP, and L6/MP lines were not significantly affected.

A case treated by face mask and Wunderer's activator, extraoral view pretreatment and posttreatment [Figures 8a and b], intraoral view before and after treatment [Figures $9 a$ and b], and cephalograms view before and after treatment [Figures 10a and b].

\section{DISCUSSION}

Skeletal Class III malocclusion can be manifested as maxillary retrognathism, mandibular prognathism, or a combination of both. "To or not to" intervene is a common dilemma faced by orthodontists and pediatric dentists when confronted with a developing Class III malocclusion. Correction of skeletal Class III malocclusion was thus initiated in the early transitional dentition to have maximum skeletal effects according to McNamara. ${ }^{[13]}$ Many appliances were used to treat skeletal Class III malocclusion. ${ }^{[14,15]}$ The present work was conducted to evaluate the effects of one of these appliances which is Wunderer activator with a face mask.

In this study, the Wunderer's activator and face mask were efficient in the correction of skeletal Class III malocclusion due to maxillary retrusion with the slight increase in vertical dimension and they improved the skeletal intermaxillary discrepancy. The results of the current study showed that Wunderer's activator with face mask can efficiently improve the dentoskeletal of patients with maxillary retrusion.

Regarding the skeletal anteroposterior effects, the results showed significant increase in the SNA angle were found. This was due to the use of face mask which provided direct constant anterior force

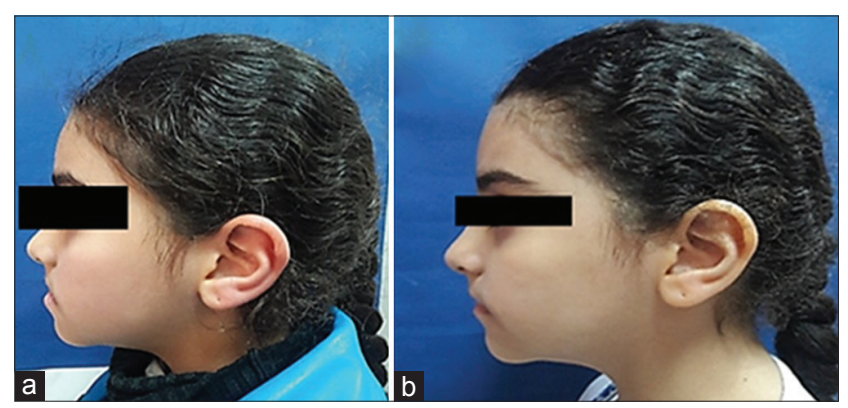

Figure 8: Extraoral profile views: (a) Before face mask and Wunderer's activator; (b) after face mask and Wunderer's activator
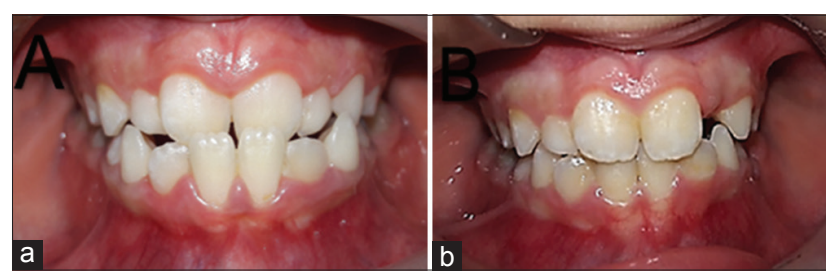

Figure 9: Intraoral profile views: (a) Before face mask and Wunderer's activator; (b) after face mask and Wunderer's activator
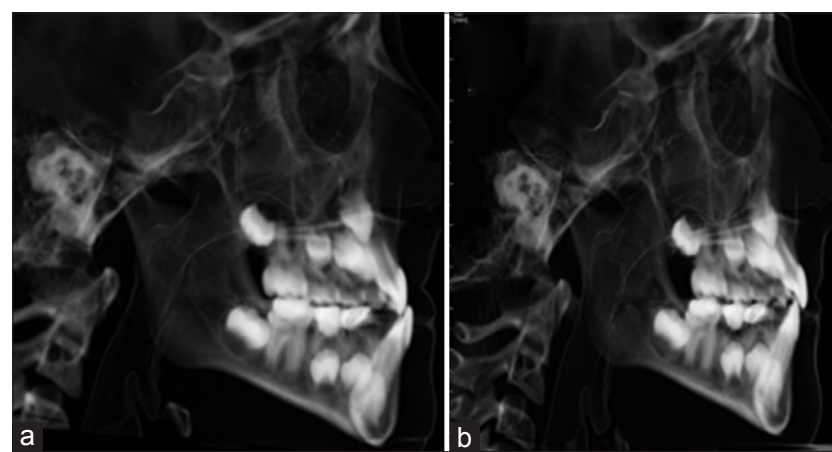

Figure 10: Cephalograms view: (a) Before face mask and Wunderer's activator. (b) After face mask and Wunderer's activator

to the maxilla, leading to anterior displacement of the maxillary sutures. The Wunderer's activator served as an intraoral appliance for the attachment of force modules as well as it created anterior force on the maxillary arch. Increase in the SNA angle was approved by McNamara, Tortop et al.; in contrast to the results of Ulgen and Firatli who utilized the Frankel III appliance had no effect on the SNA. ${ }^{[14-17]}$ Decrease the SNB angle was due to more backward displacement of the mandible with rotation, this might have been due to the chincap effect of the face mask therapy. This explains the slight increase in the facial height, also the mandible 
was held in the most retrusive position during treatment, this retrusive position led to forward and downward motion of the maxilla while mandible moved downward and backward. Thus, the ANB angle increased significantly due to the anterior movement of A point and posterior movement of B point. This outcome was in agreement with those of Arslan et al, McNamara, and Altug and Arslan. ${ }^{[14,18,19]}$ On the other hand, this result was in contrary to study conducted by Mermigos et al. and Kulbersh et al., where the ANB angle was increased by the increase in the SNA angle only. ${ }^{[20,21]}$ Regarding the vertical dimension in the current study, there was revealed slight increase in SN/OCP and SN/GOGN, respectively, this could be due to the rotation of the mandible; this might have been due to the chincap effect of the face mask therapy. ${ }^{[22]}$ The effects of the face mask are a combination of dental and skeletal changes in the mandible and the maxilla. The maxilla moved downward and forward as a result of the protractive force. As a consequence of this effect, the mandible rotated downward and backward, thus improving maxilla-mandibular relationship in the sagittal dimension. However, this led to increase in the lower facial height, also the vertical dimension can be managed in hyperdivergent growth patients by utilizing appliances with interocclusal acrylic, Wunderer activator had it, Dorothea DauschNeumann, Tubingen. ${ }^{[23,24]}$ Results of dentoalveolar changes were highly significant increased, especially upper incisors inclination (U1/SN, 1+/NA) with improvement of the overjet as result, the lower incisors (L1/MP, 1-/NB) became retroclined, due to, the force exerted by the chin cup of the face mask, also the design of this intraoral appliance depends on positioning the mandible posteriorly which in turn suggested more skeletal than dentoalveolar changes. Previous studies have approved that this backward of the mandible was a major contributing factor in establishing an improvement in anterior overjet. ${ }^{[25]}$ Dentally, the force exerted by the chin cup caused lingual inclination of the mandibular incisors, while the protractive force caused proclination in the maxillary incisors. In the present work, the position of mandibular first molar in relation to the L6-MP and the maxillary first molar in relation to the palatal plane (U6-pp) showed nonsignificant difference; in contrast with face mask and rapid maxillary expansion therapy because of the elimination of molar extrusion caused by rapid maxillary expansion. ${ }^{[26,27]}$

\section{CONCLUSION}

In the present study, it can be concluded that Wunderer's activator and a face mask were successful in improving the skeletal and dentoalveolar discrepancy, and it could be used in correction of skeletal Class III due to maxillary deficiency by restricted the growth of the mandible and enhance the advancement of the maxilla.

\section{REFERENCES}

1. Litton SF, Ackermann LV, Isaacson RJ, Shapiro BL. A genetic study of class 3 malocclusion. Am J Orthod 1970;58:565-77.

2. Rakosi T, Schilli W. Class III anomalies. A coordinated approach to skeletal, dental, and soft tissue problems. J Oral Surg 1981;39:860-70.

3. Ainsworth NJ. The Incidence of Dental Disease in the Children in Medical Research Council. Reports of the Committee for the Investigation of Dental Disease. Special Report Series; 1925. p. 97.

4. Franchi L, Baccetti T, McNamara JA. Postpubertal assessment of treatment timing for maxillary expansion and protraction therapy followed by fixed appliances. Am J Orthod Dentofacial Orthop 2004;126:555-68.

5. Wells AP, Sarver DM, Proffit WR. Long-term efficacy of reverse pull headgear therapy. Angle Orthod 2006;76:915-22.

6. Anne Mandall N, Cousley R, DiBiase A, Dyer F, Littlewood S, Mattick R, et al. Is early class III protraction facemask treatment effective? A multicentre, randomized, controlled trial: 3-year follow-up. J Orthod 2012;39:176-85.

7. Kim JH, Viana MA, Graber TM, Omerza FF, BeGole EA The effectiveness of protraction face Mask therapy: A metaanalysis. Am J Orthod Dentofacial Orthop 1999;115:675-85.

8. Roberts CA, Sabtelny JD. An American board of orthodontics case report. Use of the face mask in the treatment maxillary skeletal retrusion. Am J Orthod Dentofac Orthop 1988;93:388-94.

9. Sargod SS, Shetty N, Shabbir A. Early class III management in deciduous dentition using reverse twin block. J Ind Soc Pedo Prev Dent 2013;31:56-60.

10. Kidner G, Dibiase A. Dibiase D. Class III twin blocks: A case series. J Orthod 2003;30:197-201.

11. Ls K. Early orthodpedic class III treatment with a modified tandem appliance. J Clin Oncol 2003;37:218-23.

12. Tuncer C, Uner O. Effects of a magnetic appliance in functional class III patients. Angle Orthod 2005;75:768-77.

13. McNamara JA Jr. Treatment of children in the mixed dentition. In: Graber TM, Vanarsdall RL, editors. Orthodontics: Current Principles and Techniques. $3^{\text {rd }}$ ed. Saint Louis: Mosby; 2000. p. 545.

14. McNamara JA Jr. An orthopedic approach to the treatment of class III malocclusion in young patients. J Clin Orthod 1987;21:598-608.

15. Mitani H, Sakamoto T. Chin cap force to a Growing mandible: 
Long-term clinical reports. Angle Orthod 1984;54:93-12.

16. Tortop T, Keykubat A, Yuksel S. Facemask therapy with and without expansion. Am J Orthod Dentofacial Orthop 2007;132:467-74

17. Ulgen M, Firatli S. The effects of Frankel's function regulator on class III malocclusion. Am J Orthod Dentofac Orthop 1994;105:561-7.

18. Arslan SG, Kama JD, Baran S. Correction of a sever class III malocclusion. Am J Orthod Dentofac Orthop 2004;126:237-44

19. Altug Z, Arslan AD. Skeletal and dental effects of a mini maxillary protraction appliance. Angle Orthod 2006;76:360-8

20. Mermigos J, Full CA, Andreasen G. Protraction of the maxillofacial complex. Am J Orthod Dentofacial Orthop 1990;98:47-55

21. Kulbersh VP, Berger J, Kersten G. Effects of protraction mechanics on the mid face. Am J Orthod Dentofacial Orthop 1998;114:484-91.

22. Elnagar MH, Elshourbagy E, Ghobashy S, Khedr M,
Evansd CA. Comparative evaluation of 2 skeletal anchored maxillary protraction protocols. Am J Orthod Dentofac Orthop 2016;150:751-62.

23. Klempner L. Early treatment of skeletal class III open bite with the tandem appliance. J Clin Orthod 2011;45:308-16.

24. Deguchi T, Kuroda T, Hunt NP, Graber TM. Long-term application of chincup force alters the morphology of the dolichofacial class III mandible. Am J Orthod Dentofacial Orthop 1999;116:610-5.

25. Moullas AT, Palomo JM, Gass JR, Amberman BD, Whit J, Gustovich D. Nonsurgical treatment of apatient with a class III malocclusion. Am J Orthod Dentofac Orthop 2006;129 4 Suppl:111-8

26. Cha BK, Ngan PW. Skeletal anchorage for orthopedic correction of growing class III patients. Sem Orthod 2011;17:124-37.

27. Cevidanes L, Baccetti T, Franchi L, McNamara JA Jr., De Clerck H. Comparison of two protocols for maxillary protraction: Bone anchors versus face mask with rapid maxillary expansion. Angle Orthod 2010;80:799-806. 